\title{
DISK-WIND MODELS FOR BROAD ABSORPTION LINE QSO'S
}

\author{
T. M. YAMAMOTO \\ Institute of Astronomy, The University of Tokyo, Mitaka, Japan, \\ e-mail:yamamoto@mtk.ioa.s.u-tokyo.ac.jp
}

Broad Absorption Line (BAL) spectra of QSOs show a wealth of metal absorption lines which can help to identify enrichment mechanisms in the early universe. BAL QSOs are believed to be viewed from the side through a gas streaming outward from an accretion disk around the central source. Models for BAL QSOs based on Monte Carlo simulations are introduced, in which the gas is confined to spherical or disk geometry.

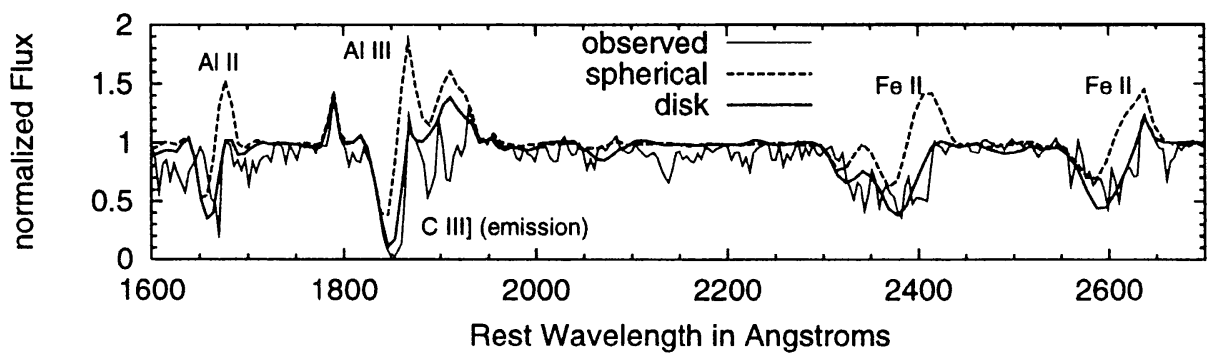

The figure shows a comparison of two synthetic spectra with an observed spectrum of the BAL QSO Q0059-2735 (thin line). The spherical model (dashed line) has too weak absorption lines and strong emission on the red wing. The emission on the red side is caused by the same phenomenon as in P-Cygni objects where we see the backward expanding shell behind the central source. In the disk model (thick line) this expanding gas can not be seen because we look from the side into the disk plane. The absorption, on the other hand, is deeper in the disk model, because each line scattering event most likely scatters the photon away from the disk plane. From this it is concluded that the disk model better reproduces the observed spectrum when compared with the spherical model spectrum.

This investigation of the outflowing gas near to the central region of QSOs shows that the appropriate geometrical structure of the absorbing gas is important for the analysis of broad absorption line regions. 